\title{
VEGFR1-TK signaling protects exacerbation of dextran sulfate sodium-induced colitis in mice
}

Tomohiro Betto ${ }^{1,2}$, Hideki Amano ${ }^{2}$, Sakiko Yamane ${ }^{1,2}$, Tomoyoshi Inoue $e^{1,2}$, Yuki Shimizu ${ }^{1,2}$, Yoshiya Ito $^{2,3}$, Tutomu Yoshida ${ }^{4}$, Masabumi Shibuya ${ }^{5}$, Wasaburo Koizumi ${ }^{1}$, Masataka Majima ${ }^{2}$

${ }^{\prime}$ Department of Gastroenterology, Kitasato university school of medicine, Japan, ${ }^{2}$ Department of Pharmacology, Kitasato University School of Medicine, Japan, ${ }^{3}$ Department of Surgery, Kitasato University School of Medicine, Japan, ${ }^{4}$ Department of Pathology, Kitasato University School of Medicine, Japan, ${ }^{5}$ Department of Research and Education, Jobu University, Japan

\section{[Background]}

Ulcerative colitis (UC) is a chronic, nonspecific inflammatory disease involving mainly the rectum and colonic mucosa. In recent reports showed that regulatory $\mathrm{T}$ cell (Treg) related to colitis but the precise mechanism is still unknown. We had reported that VEGFR1 tyrosine kinase (TK) signaling facilitates gastric ulcer healing. Based on previous study, we hypothesized VEGFR1-TK signaling related to the colonic mucosal healing process by accumulation of Treg.

[Methods]

Male 8-10 weeks of age, C57BL/6 mice (Wild type; WT) and VEGFR1TK deficient mice (VEGFR1TK -/-) were used. DSS colitis was induced by given 2.0\% DSS dissolved in tap water ad libitum for 7days. Disease activity index (DAI) was evaluated by scoring changes in body weight loss, stool consistency and bloody stool. The size of mucosal damage was estimated by hematoxylin-eosin staining. Angiogenesis was estimated by the expression of CD31 by real time PCR and immunohistochemical analysis. The expressions of inflammatory and anti-inflammatory cytokines in inflamed mucosa were estimated by real time PCR. Furthermore, the number of Foxp3+Treg in the inflamed mucosa was estimated by immunohistochemical analysis.

[Results]

Body weight gain was significantly decreased in VEGFR1TK-/- on day 12 and 14. DAI score was significantly high in VEGFR1TK-/- compared to WT on day 14. The mucosal healing was delayed in VEGFR1TK-/-. Furthermore, the expression of mRNA level of CD31 and the number of CD31+ vessels were suppressed in VEGFR1TK-/-. The expression of TGF- $\beta$, IL-6, IL-10, EGF and Foxp3 were suppressed in VEGFR1TK-/- compared to WT. Moreover, the number of accumulated Foxp3+ VEGFR1+Treg in inflamed mucosa was decreased in VEGFR1TK-/- compared to WT.

[Conclusion]

These results suggested that VEGFR1-TK signaling induced mucosal healing by accumulation of Foxp3+VEGFR1+Treg in DSS induced colitis. 